\title{
The Application of Autonomous Robots Group for Monitoring of Oil Platform Condition
}

\author{
Ivan Vasilyev ${ }^{1}$, Ekaterina Smirnova ${ }^{1}$, Alla Koshurina ${ }^{2}$, Eugene Kadilenko ${ }^{2}$ and Maxim Krasheninnikov ${ }^{2}$ \\ ${ }^{1} 194064$, St. Petersburg, Tihoreckij prospect, 21, CNII RTK, Russia \\ ${ }^{2} 603950$, Russia, Nizhny Novgorod, Minina street, 24, NNSTU named after R.E. Alekseev
}

\begin{abstract}
The main purpose of this article is the review of abilities of autonomous robots group application for monitoring of oil platform condition. The article contains functions performed by the robots group; the composition of the robots group; robots' system firmware and possible robots' location on an oil platform.
\end{abstract}

Keywords-robots; group interaction; rescue operations; oil platform; computer vision system

\section{INTRODUCTION}

The organization of rescue operations by means of autonomous robots is the extremely difficult issue. Lately, amount of disasters and, as result, amount of human victims has been increasing. One of causes of this situation is increase of oil and gas extraction in offshore and Arctic regions.

However, there is the increase of practical experience of robotic devices application for security of human work activities. The particular case is the application of robots group. The application of robots interacting among each other can reduce the time of rescue operations.

The authors Karpov V.Je. and Dobrynin D.A descripted a scheme of command work of underwater robots in a port station [1]. Several autonomous underwater robots (AUR) patrol the underwater space of the port station. Every robot has own trajectory of patrolling. AUR can connect with each other and with a control centre (CC).

Every AUR has equipment for detection of underwater objects and equipment for active sounding for object identification (mines; diver; safe object and other). If an AUR (further the AUR-initiator) detects an unknown object, the robot will make the command (use the protocol of common intentions) for more detailed exploration of the object with other members of the group. Other AURs take obligations and agreements and take decision of zones and angles of the object photography. Hereafter, involved AUR autonomously make own decisions and transfer information to the AUR-initiator.

The results of the experiment of the robots group network interaction for group searching were evaluated in the experimental work of authors O. Caypunar and V. Gazi [2]. The simulation and the experimental testing were carried out in the work. It gave results that are more objective. The performance of two strategies of searching ("spiral search" and random search) was measured for different communicating robots and different amount of the robots. It was found out that the performance increases with the increase of the communicating robots amount. The performance of 6 robots is higher than the performance of 3 robots. The performance of 3 robots is higher that of the one robot. The results of the experiment showed that the communication range increase leads to the performance increase for the reviewed scenarios of searching. The performance is maximum when the communication range is equal or more than dimensions of the group. However, the performance does not increase with further increase of the communication range. Thereby, for the performance increase doesn't need global connection. Other words, it is not necessary to cover the whole searching area.

The advantages of robots group application are [3]:

- biggest operating range;

- higher amount of performing functions;

- durability of a robots group is higher than durability of one robot

- higher likelihood of successfully mission completion.

Therefore, a group of robots can perform such difficult tasks (exploration and sounding inaccessible areas; assembly of difficult constructions; support military operations and other) efficiently. However, new issues of organization of group control and communication appear.

\section{FUNCTIONS, COMPOSITION AND SYSTEM FIRMWARE OF ROBOTS GROUP}

The one of most important tasks where a robots group can be used is monitoring of condition of a technical object for prevention of an accident on an oil platform. It is also necessary for rescue operation organization in case of an accident [4].

A robots group can perform next tasks:

- monitoring of oil platform condition;

- searching for ways of evacuation;

- monitoring of condition of constructions during an accident;

- scouting of safe and convenient places for people placement;

- accompaniment of human for reaching of safe places. 
A robots group should be maximum excessive for trouble proof tasks performance. The robots group should be divided for subgroups, which can replace each other for recharging and maintenance during an operation. Likewise, it's necessary to have robots which don't participate in the operation, but are ready to replace destroyed robots during the operation.

If robots, which monitor a situation around an oil platform, detect emergency, they will give a signal to other members of the group. Then the group gets ready to start rescue operation. Other robots (which didn't participate in monitoring and scouting) should be got ready for the operation after testing.

Robots-scouts will continue the monitoring until they get the command to go away from an oil platform. They will get the command if:

- there is a signal to stop the operation

- all people are rescued

- there is a signal to stop the operation, if the rescue of other people is impossible;

- other criteria

The robots during the operation continuously keep track people placement on the oil platform, estimate the risk level and ways for evacuation.

Robots are actively involved in creation of safe routes and evacuation of people from the destroying platform. Robots should have fire and smoke sensors and must be able to detect unstable and damaged structure, and determine the likelihood of structural collapse.

In the search for a safe escape route robots should analyze possibility of moving on chosen route for people. Because, there is the situation when movement of people will not be possible, while to move the robot, this may not be a serious obstacle (for example, because of the heavy smoke, high temperatures or surfaces of movement and others). Therefore, every robot must be equipped with appropriate sensors.

Each robot must be equipped with computer vision system to carry out functions of observation and movement. The composition of the computer vision system can be different for each robot separately, depending on the priority of functions performed by a robot, and the dimensions of a robot.

In general, each robot, as a minimum, should have a plurality of laser or ultrasonic range finders and a television stereo system. Also, the robot's computer vision system can be supplemented with a laser scanner system with structured lighting, additional cameras with the extra-short focus or the long focus lens, a thermal imager and an active-pulse night vision devices. It depends on the functions and processing power of a robot.

In addition, each robot in the group should share information obtained from the vision system for creation of a general most complete and accurate map of surrounding space.

\section{LOCATION OF THE GROUP ON THE OIL PLATFORM}

Robots-scouts should move freely on the platform and on a water-ice surface near of the platform. At the same time, the robot must not interfere with works of the equipment and personnel. Therefore, there is an issue, how to get timely and full information about the platform condition and do not interfere to the work (Figure 1). On the figure below, robotsscouts are indicated by green arrow.

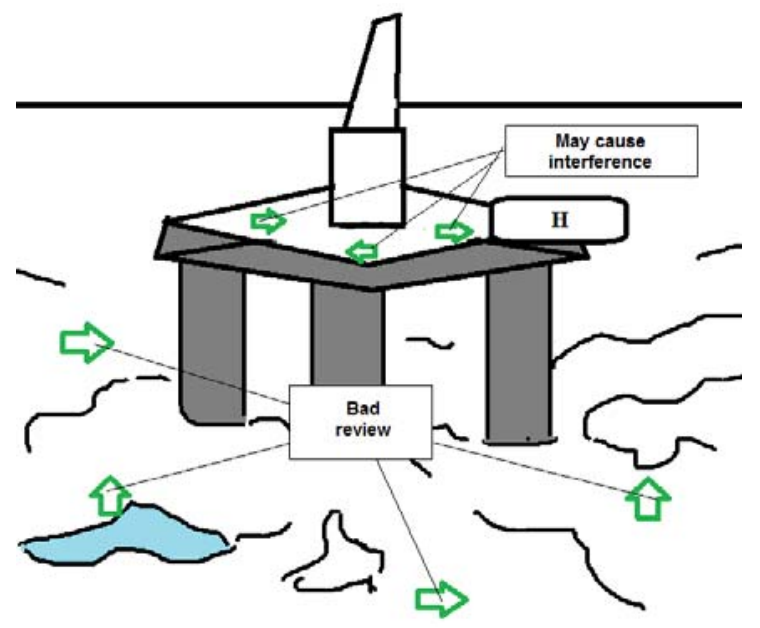

FIGURE I.CAPTION POSSIBLE PLACEMENT OF THE ROBOTS ON THE PLATFORM AND AROUND IT

Some robots can be located at specific sites of the oil platform not interfere with the people and equipment (Figure 2). On the picture, green pentagons show robots-inspectors.

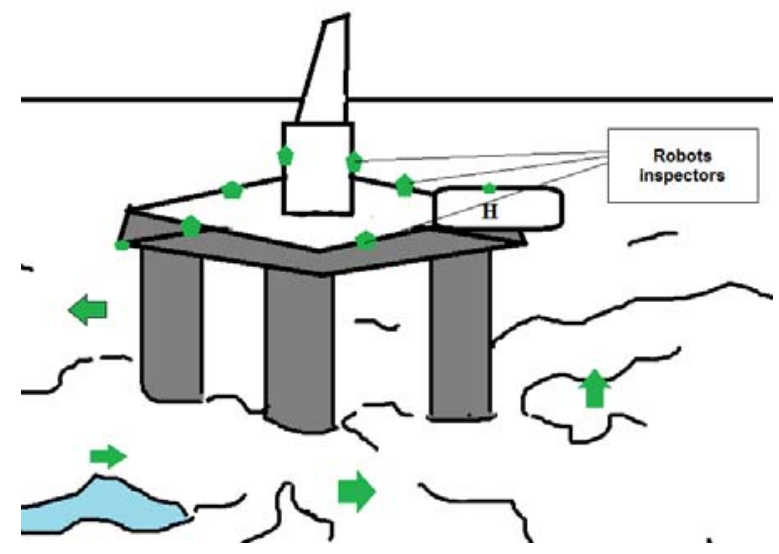

FIGURE II. PLACING ON THE PLATFORM STATIONARY ROBOTS-INSPECTORS

However, these robots will not be able to participate actively in the process of saving people: creation and updating maps, scouting, and will be limited in their areas of visibility. In addition, such robots will be destroyed in case of structural collapse of the platform. Therefore, the whole group will be deprived of information on this area of the platform, before robot-scout arrival. In this situation, there will be no actual information about platform condition and availability and condition of the people on the most dangerous zone of the platform. Therefore, in case of failure of one of these robots- 
inspectors the mobile robot-scout should go to the area of accident start, which was assigned for him.

Another solution can be achieved by modification of oil platform for the movement of mobile robots-scouts. In addition, the oil platform can be equipped with lift system for mobile robots-scouts on the platform. The constructive part of the platform, created especially for the movement of robotsscouts and not intended for the movement of people should be connected with all areas of the oil platform, should not prevent the descent of robots in traffic areas. Descent and lift of robots with special lanes should occur freely throughout the portion of the band, and the selected for robot motion band should not prevent for evacuation and movement of people, their work and the work mechanisms of the platform (Figure 3).

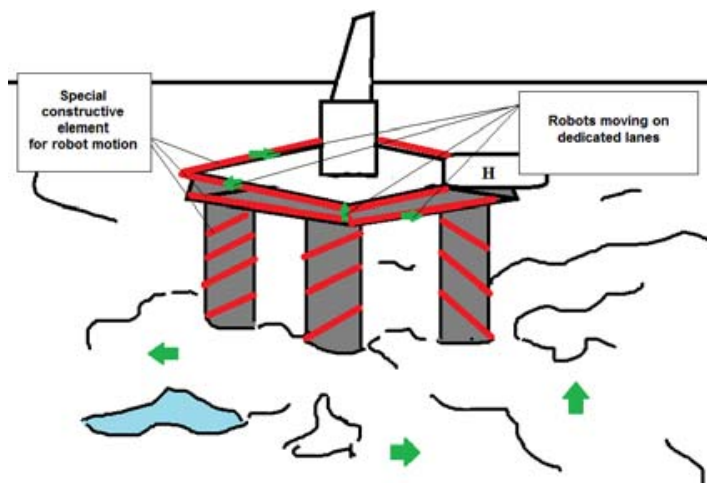

FIGURE III. ROBOTS-SCOUTS, MOVING IN A DEDICATED LANE

\section{CONCLUSION}

The best solution may be found in a synthesis of all the proposed methods. Some robots-inspectors are always located on the oil platform on fixed and predetermined positions, but they are not rigidly fixed and can be set at any moment in motion for people rescue operation, mapping, scouting and exploration of environment of the robot.

In the case of contingency, a robot must act in accordance with a prescribed script, for example, to leave the area of danger. At the same time, the location of people on the platform should be taken into account during the withdrawal from the danger zone, to avoid blind spots in areas of the oil platform where people can be located. In this case, will be better if the robot stay in the danger zone to provide information of this area, in case if people are there.

Our scientific collective have developed software systems, allowing for realizing: a mathematical modeling of robot members of the group (№2015662332); mathematical modeling of onboard systems control robots (№2015662333). These software packages may also be useful for functioning of the group of autonomous robots for monitoring the oil platform.

These works were carried out in NNSTU named after R.E. Alekseev, with the financial support of the Ministry of Education and Science under the "Research and development on priority directions of scientific-technological complex of
Russia for 2014-2020", the unique identifier of the project: RFMEFI57414X0055.

\section{REFERENCES}

[1] Karpov V.Je., Dobrynin D.A. "Modeling some of the simplest forms of behavior from conditioned reflexes to the inductive classification" // Trudy I mezhdunarodnoj konferencii «Sistemnyj analiz i informacionnye tehnologii» SAIT-2005 (12-16 sentjabrja 2005 g., Pereslavl'-Zalesskij, Rossija), M.: KomKniga, T.1, str. 188-193

[2] O. Caypunar, V. Gazi, B. Tavli, E. Cervera, U. Witkowski, and J. Penders, "Experimental study on the effect of communication range on cooperative robotic search in complex environments", in Proceedings of the International Conference on Robot Communication and Coordination, Odense, Denmark, April 2009

[3] Vasilyev, A. Kashourina, M. Krasheninnikov, E. Smirnova. Use mobile robots groups for rescue missions in extreme climatic conditions // Annals of 25th DAAAM International Symposium on Intelligent Manufacturing and Automation, DAAAM 2014

[4] A. Koshurina, M. Krasheninnikov, S. Kaniskin, R. Dorofeev, V. Obrezkova, "The Development of the Intellectual System Optimization Search Operations", Applied Mechanics and Materials, Vol. 763, pp. 164-169, May. 2015 